\title{
Article
}

Mycosphere

\section{Three species of Neofusicoccum (Botryosphaeriaceae, Botryosphaeriales) associated with woody plants from southern China}

\author{
Zhang $\mathrm{M}^{1,2}$, Lin $\mathrm{S}^{1,2}$, He $\mathrm{W}^{2, *}$ and Zhang $\mathrm{Y}^{1, *}$ \\ ${ }^{1}$ Institute of Microbiology, P.O. Box 61, Beijing Forestry University, Beijing 100083, PR China. \\ ${ }^{2}$ Beijing Key Laboratory for Forest Pest Control, Beijing Forestry University, Beijing 100083, PR China.
}

Zhang M, Lin S, He W, Zhang Y 2017 - Three species of Neofusicoccum (Botryosphaeriaceae, Botryosphaeriales) associated with woody plants from Southern China. Mycosphere 8(2), 797-808, Doi 10.5943/mycosphere/8/2/4

\begin{abstract}
Two new species, namely $N$. sinense and $N$. illicii, collected from Guizhou and Guangxi provinces in China, are described and illustrated. Phylogenetic analysis based on combined ITS, tefl- $\alpha$ and TUB loci supported their separation from other reported species of Neofusicoccum. Morphologically, the relatively large conidia of $N$. illicii, which become 1-3-septate and pale yellow when aged, can be distinguishable from all other reported species of Neofusicoccum. Phylogenetically, $N$. sinense is closely related to $N$. brasiliense, $N$. grevilleae and $N$. kwambonambiense. The smaller conidia of $N$. sinense, which have lower L/W ratio and become $1-$ 2-septate when aged, differ from the other three species. Neofusicoccum mangiferae was isolated from the dieback symptoms of mango in Guangdong Province.
\end{abstract}

Key words - Asia - endophytes - Morphology- Taxonomy

\section{Introduction}

Neofusicoccum Crous, Slippers \& A.J.L. Phillips was introduced by Crous et al. (2006) for species that are morphologically similar to, but phylogenetically distinct from Botryosphaeria species, which are commonly associated with numerous woody hosts world-wide (Arx 1987, Phillips et al. 2008). Some species of Neofusicoccum are reported to produce a Dichomera synanamorph, which may serve as a distinguishing characteristic from Botryosphaeria (Crous et al. 2006). There are 34 epithets included in Neofusicoccum according to Index Fungorum (2017), although most species previously described under Fusicoccum Corda are likely to reside in Neofusicoccum (Crous et al. 2006). Species of Neofusicoccum are differentiated on the basis of conidial dimensions, pigmentation of the culture media and DNA sequence data, although the taxonomic significance of some of the morphological characters has been questioned (Phillips et al. 2008), and patterns of septation and coloration in aged conidia discharged from pycnidia was regarded as a useful morphological feature to distinguish some species in Neofusicoccum and other genera of Botryosphaeriaceae (Slippers et al. 2004, Abdollahzadeh et al. 2013, Dissanayake et al. 2016).

In the course of an ongoing survey of biodiversity of Botryosphaeriaceae in China initiated in 2014, three Botryosphaeriaceous species that morphologically fit within Neofusicoccum were isolated from dieback symptoms as well as healthy tissues of some woody plants. The generic status of these isolates in Neofusicoccum was supported by their morphology and ITS, tefl- $\alpha$ and 
TUB $n u$ DNA sequences phylogenetic analysis. Based on the combination of subtle morphological and molecular differences, two new species together with $N$. mangiferae are reported here.

\section{Materials \& Methods}

\section{Fungal isolation and morphology}

Fresh material was collected from dieback symptoms and healthy tissues of some common tree species were collected from Guizhou, Guangdong and Guangxi provinces in Southern China from 2012 to 2016. Isolations were made from dead, diseased or healthy tissue of woody plants, and transferred to malt extract agar (MEA), and put in the ambient temperatures (about $28{ }^{\circ} \mathrm{C}$ ) in the dark to establish colony characteristics, then transferred to synthetic nutrient-poor agar (SNA) with sterilized pine needles for three weeks to induce sporulation. Microscopic observations were made from material mounted in water. Photomicrographs were taken with a Nikon Coolpix 995 digital camera on a Leitz Orthoplan microscope. Measurements of conidia and conidiogenous cells were made from water mounts. Fungal isolates have been deposited at China General Microbiological Culture Collection Center (CGMCC) and herbarium specimens at the Mycological Herbarium of the Institute of Microbiology Chinese Academy of Sciences (HMAS).

\section{DNA extraction, PCR amplification and sequencing}

Colonies for DNA extraction were grown on MEA plates in darkness at $28{ }^{\circ} \mathrm{C}$ for $4-6$ days until they completely covered the agar surface. DNA was extracted from the mycelium with CTAB plant genome DNA fast extraction kit (Aidlab Biotechnologies Co, Ltd, Beijing, China). The internal transcribed spacer of rDNA (ITS) was amplified and sequenced with primers ITS1 and ITS4 (White et al. 1990). Part of the translation elongation factor-1a (tefl- $\alpha$ ) was amplified and sequenced with primers EF1-688F and EF1-1251R (Alves et al. 2008) and part of the TUB gene was amplified and sequenced with primers Bt2a and Bt2b (Glass \& Donaldson 1995). DNA amplification and sequencing followed the protocol of Zhang et al. (2009).

\section{Sequence alignment and phylogenetic analysis}

The combined ITS, tefl- $\alpha$ and TUB $n u$ DNA sequence dataset was used to infer the phylogenetic relationships among the new species and other reported species of Neofusicoccum. Sequences generated were analyzed with other sequences obtained from GenBank (Table 1). A multiple alignment was done in MEGA v. 6 (Tamura et al. 2013). Prior to the phylogenetic analysis, ambiguous regions at the start and the end of the sequences were deleted and gaps manually adjusted to optimize alignment. For Bayesian analysis, the best-fit model of nucleotide evolution (GTR+I+G) was selected using the Akaike information criterion (AIC; Posada \& Buckley 2004) in MrModeltest 2.3. The metropolis-coupled Markov Chain Monte Carlo (MCMCMC) approach was used to calculate posterior probabilities (Ronquist \& Huelsenbeck 2003). A preliminary Bayesian inference (BI) analysis using MrBayes revealed that the MCMC (Huelsenbeck \& Ronquist 2001) steady state was reached after less than 2,260,000 generations (the average standard deviation of split frequencies was constantly below 0.01). A conservative burn-in of 22,600 trees was chosen and a full analysis of 10,000,000 generations was carried out with sampling every 100 generations. Maximum Parsimony (MP) analysis was conducted in PAUP v. 4.0b10 (Swofford 2002). Trees were inferred using the heuristic search option with 1,000 random sequence additions and tree-bisection-reconnection (TBR) as the branch-swapping algorithm and gaps were treated as missing data. Maxtrees were set to 50,000, branches of zero length were collapsed and all multiple parsimonious trees were saved. Trees were viewed in TREEVIEW (Page 1996). Nucleotide sequences generated in this paper were deposited in GenBank. Trees and alignments were deposited in TreeBase with study ID S20904. 


\section{Results}

\section{Phylogenetic analyses}

The combined ITS, tefl $-\alpha$ and TUB $n u$ DNA sequence dataset consisted of 73 strains and 1243 characters in the MP analysis. Of the included bases, 270 sites $(21.7 \%)$ were parsimonyinformative. A heuristic search with random addition of taxa (1,000 replicates) treating gaps as missing characters generated 5000 equally parsimonious trees, each with similar topology (figures not shown). A single parsimonious tree $(\mathrm{TL}=629, \mathrm{CI}=0.676, \mathrm{RI}=0.872, \mathrm{RC}=0.589, \mathrm{HI}=$ 0.324 ) is shown in Fig. 1. Bayesian posterior probabilities (PP) support equal to or greater than 70 $\%$ from Bayesian analysis and maximum parsimony (MP) support values greater than $60 \%$ are shown with Bayesian PP followed by MP bootstrap (PP/MP) values at the nodes (Fig. 1). This tree resolved 37 clades corresponding to 35 known and two previously unknown species. Therefore, two new species are introduced here.

\section{Taxonomy}

Neofusicoccum illicii Y. Zhang ter., M. Zhang sp. nov.

Fig. 2

MycoBank MB 819397; Facesoffungi number: FoF 02822.

Etymology - named after the host from which it was isolated, Illicium verum.

Ascomata not observed. Conidiomata stromatic, produced on pine needles on SNA within 14 $\mathrm{d}$, solitary or in groups covered by mycelium, dark brown to black, 2/3-3/4 erumpent, ellipsoidal or spherical, 250-350 $\mu \mathrm{m}$ diam. Paraphyses not observed. Conidiophores reduced to conidiogenous cells. Conidiogenous cells holoblastic, hyaline, smooth-walled, cylindrical or claviform, sometimes forming a periclinal thickening, (9-) 10.5-13 (-16) $\times(2-)$ 2.6-3.1 (-3.5) $\mu \mathrm{m}$. Conidia hyaline, thin walled, granular cytoplasm, broadly to narrowly fusiform, or nearly cylindrical, sometimes slightly curved, base truncate or sometimes node-like, initially non-septate, subsequently becoming 1-3septate and pale yellow, (22-) 23.7-27.1 (-30) $\times(5-)$ 6.1-7.9 (-9) $\mu \mathrm{m}$ (av. of 30 conidia $=25.4 \pm$ $1.2 \times 7 \pm 0.8 \mu \mathrm{m}, \mathrm{L} / \mathrm{W}$ ratio $=3.6)$, usually constricted at the main septum. Spermatia not observed.

Cultural characteristics - Colonies on MEA grey-white (surface) and buff to light primrose (reverse), sometimes not reaching the edge of the plate, with a dense mat of aerial mycelium, covering a $30 \mathrm{~mm}$ Petri dish in 3 days, the margin crenulated irregularly.

Specimens examined - CHINA, Guangxi province, from healthy tissue of Illicium verum, 7 Sept. 2012, L. Wang (holotype: HMAS 266205; cultures ex-holotype: CGMCC 3.18310; CGMCC 3.18311; CGMCC 3.18312; CGMCC 3.18313).

Notes - Phylogenetically, N. illicii is sibling to all other species of Neofusicoccum, and basal to the clade comprising $N$. algeriense, $N$. batangarum, $N$. brasillense, $N$. cordaticola, $N$. grevilleae, $N$. kwambonambiense, $N$. occulatum, N. parvum, N. ribis, $N$. sinense and N. umdonicola (Fig. 1). Morphologically, the large conidia of $N$. illicii differentiate this species from $N$. algeriense, $N$. batangarum, $N$. occulatum, $N$. parvum, $N$. umdonicola and $N$. sinense (Crous et al. 2006, Pavlic et al. 2008, Begoude et al. 2010, Sakalidis et al. 2011, Berraf-Tebbal et al. 2014). The septate aged conidia with $\mathrm{L} / \mathrm{W}$ radio of $N$. illicii are most comparable with $N$. grevilleae, while the small condiomata $(<200 \mu \mathrm{m})$ of $N$. grevilleae (Crous et al. 2011) differentiate it from $N$. illicii.

Neofusicoccum sinense Y. Zhang ter., M. Zhang sp. nov.

Fig. 3

MycoBank MB 819396; Facesoffungi number: FoF 02821.

Etymology - The epithet sinense refers to China (from Latin sinensia = China), the country from which it is described.

Ascomata not observed. Conidiomata stromatic, produced on pine needles within 1-2 week, solitary or botryose covered with white mycelium, dark brown to black, initially immersed, 1/2 erumpent through the pine needles at maturity, spherical or elliptical or with central, black ostioles, 200 to $350 \mu \mathrm{m}$ diam. Paraphyses not observed. Conidiophore not observed. Conidiogenous cells not observed. Conidia hyaline, thin walled, granular cytoplasm, narrowly to broadly fusiform, initially non-septate, subsequently becoming 1-2-septate, constricted at the septum, apex rounded, 
with somewhat truncate base or sometimes curved node-like, (15.2-) 17.6-20.4 (-23) × (6.9-) 7.4$8(-9) \mu \mathrm{m}$ (av. of 20 conidia $=18.7 \pm 1.5 \times 7.7 \pm 0.9 \mu \mathrm{m}, \mathrm{L} / \mathrm{W}$ ratio $=2.4)$. Spermatogenous cells hyaline, slimy cylindrical, smooth-walled and radiating divergent to the surrounding, (10-) 12-13.5 $(-15) \times 3-5.2 \mu \mathrm{m}$, inflated near the base and somewhat tapering upward, apex usually attached to spermatia which is going to fall off. Spermatia hyaline, cylindrical, aseptate, sometimes with arc bending, $5 \times 3 \mu \mathrm{m}$.

Cultural characteristics - Colonies on MEA iron-grey (surface) and olivaceous- grey (reverse) with extensive grey aerial mycelium, and smooth margins, attaining a radius of $20 \mathrm{~mm}$ after 3 days in darkness at $28^{\circ} \mathrm{C}$, aerial mycelium growing upward like conical antenna and eventually form the bowl colony about 10 days, tapered tip and part with slowly atrophy then flattened with tufts, its color transition from white to grey or ash grey after 25 days.

Specimens examined - CHINA, Guizhou Province, Huangping County, Fengxiangzhai (altitude: 1,000 m), from branch of unknown dead woody plant, 20 Feb. 2016, J.J. Gan (holotype: HMAS 255209; culture ex-holotype: CGMCC 3.18315).

Notes - Phylogenetically, N. sinense is sibling to other species of Neofusicoccum, while closely related to $N$. brasillense, $N$. grevilleae and $N$. kwambonambiense (Fig. 1). Morphologically, the conidia of $N$. sinense are initially non-septate but subsequently become 1-2-septate. Neofusicoccum brasiliense failed to sporulate in culture, thus its description was based solely on molecular data (Marques et al. 2013). The small-sized conidiomata $(<200 \mu \mathrm{m})$, larger conidia $(20$ $32 \times 6-10 \mu \mathrm{m})$ and the phialidic conidiogenous cells of $N$. grevilleae are distinguishable from those of $N$. sinense. The presence of spermatia, smaller conidia and lower L/W ratio of $N$. sinense $(18.7 \times$ $7.7 \mu \mathrm{m}, \mathrm{L} / \mathrm{W}=2.4)$ are distinguishable from those $N$. kwambonambiense $(22.3 \times 6.3 \mu \mathrm{m}, \mathrm{L} / \mathrm{W}=$ 3.6). Thus, a new species, $N$. sinense, is introduced here.

Neofusicoccum mangiferae (Syd. \& P. Syd.) Crous, Slippers \& A.J.L. Phillips, Stud. Mycol. 55: 248 (2006)

三Dothiorella mangiferae Syd. \& P. Syd., Annls mycol. 14(3/4): 192 (1916)

Specimens examined - CHINA, Guangdong province, Yangchun, Kongtong mountain, Mangifera indica, 23 Jan. 2016, Z.P. Dou \& Z.C. Liu (CGMCC 3.18314).

\section{Discussion}

A few species of Neofusicoccum had been reported in China as plant pathogens. For instance, $N$. parvum causes gummosis of mango ( $\mathrm{Li}$ et al. 2014), stem die-back of blueberries ( $\mathrm{Yu}$ et al. 2013) and canker of Juglans regia seedlings (Yu et al. 2015). Neofusicoccum vitifusiforme causes blueberry blight (Kong et al. 2010), and N. mangiferae causes grapevine dieback in Henan and Anhui Provinces in China (Dissanayake et al. 2015). In this study, three species of Neofusicoccum were isolated from subtropical regions in China, and two of which, namely N. illicii and N. sinense, are new to science. Neofusicoccum illicii was isolated from Illicium verum as endophyte in Guangxi Province in China, while $N$. sinense was isolated from an unidentified dead woody plant as saprophyte in Guizhou province.

Botryosphaeriacous fungi are among the most severe pathogens that affect mango trees and fruits (Slippers et al. 2005). Neofusicoccum mangiferae has been widely reported as a pathogen of Mangifera indica worldwide wherever mangoes are grown, for instance in India (Sydow \& Sydow 1916), Australia (Johnson et al. 1991, Johnson 1992, Slippers et al. 2005), United States (Mitra \& Baldwin 1997), Puerto Rico (Serrato-Diaz et al. 2014) and China (this study). Besides $N$. mangiferae, other species of Neofusicoccum, such as $N$. australe, $N$. brasilense, $N$. mediterraneum and $N$. parvum have also been reported as prevalent pathogens of mango causing fruit stem-end rot, dieback, gummosis and blossom blight (Slippers et al. 2005, Adesemoye \& Eskalen 2011, Abdollahzadeh et al. 2013, Barradas et al. 2013, Ismail et al. 2013, Li et al. 2013, 2014, Marques et al. 2013, Lopes et al. 2014, Krishnapillai et al. 2015). 
Table 1 Details of Neofusicoccum strains and species considered in this study (newly generated sequences are indicated in bold).

\begin{tabular}{|c|c|c|c|c|c|c|}
\hline \multirow[t]{2}{*}{ Species } & \multirow[t]{2}{*}{ Strain } & \multirow[t]{2}{*}{ Host } & \multirow[t]{2}{*}{ Origin } & \multicolumn{3}{|c|}{ GenBank Accession no. } \\
\hline & & & & ITS & tef1-a & $T U B$ \\
\hline \multirow{2}{*}{$\begin{array}{l}\text { Neofusicoccum } \\
\text { algeriense }\end{array}$} & CBS 137504 & Vitis vinifera & Algeria & KJ657702 & KJ657715 & KX505915 \\
\hline & CAA 322 & Eucalyptus globulus & - & KX505906 & KX505894 & KX505916 \\
\hline \multirow[t]{2}{*}{ N. andinum } & CBS 117453 & Eucalyptus sp. & Venezuela & AY693976 & AY693977 & KX464923 \\
\hline & CBS 117452 & Eucalyptus sp. & Venezuela & DQ306263 & DQ306264 & KX464922 \\
\hline \multirow[t]{2}{*}{ N. arbuti } & CBS 116131 & Arbutus menziesii & USA & AY819720 & KF531792 & KF531793 \\
\hline & CBS 117090 & Arbutus menziesii & USA & DQ306263 & KF531791 & KF531794 \\
\hline \multirow[t]{2}{*}{ N. australe } & CMW 6837 & Acacia sp. & Australia & AY339262 & AY339270 & AY339254 \\
\hline & CMW 6853 & Sequiadendron sp. & Australia & AY339263 & AY339271 & AY339255 \\
\hline \multirow[t]{2}{*}{ N. batangarum } & CBS 124924 & Terminalia catappa & Africa & FJ900607 & FJ900653 & FJ900634 \\
\hline & CBS 124923 & Terminalia catappa & Africa & FJ900608 & FJ900654 & FJ900635 \\
\hline \multirow[t]{2}{*}{ N. brasiliense } & CMM 1338 & Mangifera indica & Brazil & JX513630 & JX513610 & KC794031 \\
\hline & CMM 1269 & Mangifera indica & Brazil & JX513629 & JX513609 & KC794032 \\
\hline \multirow[t]{2}{*}{ N. buxi } & CBS 116.75 & Buxus sempervirens & Sweden & KX464165 & KX464678 & - \\
\hline & CBS 113714 & Buxus sempervirens & France & KX464164 & KX464677 & KX464954 \\
\hline \multirow[t]{2}{*}{ N. cordaticola } & CBS 123634 & Syzygium cordatum & South Africa & EU821898 & EU821868 & EU821838 \\
\hline & CBS 123635 & Syzygium cordatum & South Africa & EU821903 & EU821873 & EU821843 \\
\hline \multirow[t]{2}{*}{ N. corticosae } & CBS 120081 & Eucalyptus corticosa & $\begin{array}{l}\text { New South } \\
\text { Wales }\end{array}$ & DQ923533 & KX464682 & KX464958 \\
\hline & CBS 118099 & Eucalyptus camaldulensis & Australia & KX464168 & KX464681 & KX464957 \\
\hline \multirow[t]{2}{*}{ N. cryptoaustrale } & CMW 23785 & Eucalyptus sp. & South Africa & FJ752742 & FJ752713 & FJ752756 \\
\hline & CMW 23786 & Eucalyptus sp. & South Africa & FJ752744 & FJ752714 & FJ752753 \\
\hline \multirow[t]{2}{*}{ N. eucalypticola } & CBS 115766 & Eucalyptus rossii & Australia & AY615143 & AY615135 & AY615127 \\
\hline & CBS 115679 & Eucalyptus rossii & Australia & AY615141 & AY615133 & AY615125 \\
\hline \multirow[t]{2}{*}{ N. eucalyptorum } & CMW 10125 & Eucalyptus grandis & South Africa & AF283686 & AY236891 & AY236920 \\
\hline & CMW 10126 & Eucalyptus grandis & South Africa & AF283687 & AY236892 & AY236921 \\
\hline N. grevilleae & CBS 129518 & Grevillea aurea & Australia & JF951137 & - & - \\
\hline
\end{tabular}




\begin{tabular}{|c|c|c|c|c|c|c|}
\hline \multirow[t]{2}{*}{ N. hellenicum } & CERC 1947 & Pistachia vera & Greece & KP217053 & KP217061 & KP217069 \\
\hline & CERC 1948 & Pistachia vera & Greece & KP217054 & KP217062 & KP217070 \\
\hline N. illicii & CGMCC 3.18310 & Illicium verum & Guangxi, China & KY350149 & - & KY350155 \\
\hline N. illicii & CGMCC 3.18311 & Illicium verum & Guangxi, China & KY350150 & KY817756 & KY350156 \\
\hline N. illicii & CGMCC 3.18312 & Illicium verum & Guangxi, China & KY350151 & KY817757 & KY350157 \\
\hline N. illicii & CGMCC 3.18313 & Illicium verum & Guangxi, China & KY350152 & KY817758 & KY350158 \\
\hline \multirow[t]{2}{*}{ N. kwambonambiense } & CBS 123639 & Syzygium cordatum & South Africa & EU821900 & EU821870 & EU821840 \\
\hline & CBS 123641 & Syzygium cordatum & South Africa & EU821919 & EU821889 & EU821859 \\
\hline \multirow[t]{2}{*}{ N. lumnitzerae } & CBS 139674 & Lumnitzera racemosa & South Africa & KP860881 & KP860724 & KP860801 \\
\hline & CBS 139675 & Lumnitzera racemosa & South Africa & KP860882 & KP860725 & KP860803 \\
\hline \multirow[t]{2}{*}{ N. luteum } & CBS 110299 & Vitis vinifera & Portugal & AY259091 & AY573217 & DQ458848 \\
\hline & CBS 110497 & Vitis vinifera & Portugal & EU673311 & EU673277 & EU673092 \\
\hline \multirow[t]{2}{*}{ N. macroclavatum } & WAC 12444 & Eucalyptus globulus & Australia & DQ093196 & DQ093217 & DQ093206 \\
\hline & WAC 12446 & Eucalyptus globulus & Australia & DQ093197 & DQ093218 & DQ093208 \\
\hline \multirow[t]{3}{*}{ N. mangiferae } & CBS 118531 & Mangifera indica & Australia & AY615185 & DQ093221 & AY615172 \\
\hline & CBS 118532 & Mangifera indica & Australia & AY615186 & DQ093220 & AY615173 \\
\hline & CGMCC 3.18314 & Mangifera indica & $\begin{array}{l}\text { Guangdong, } \\
\text { China }\end{array}$ & KY350153 & KY817759 & KY350159 \\
\hline \multirow[t]{2}{*}{ N. mangroviorum } & CMW 41365 & Avicennia marina & South Africa & KP860859 & KP860702 & KP860779 \\
\hline & CMW 42481 & Avicennia marina & South Africa & KP860848 & KP860692 & KP860770 \\
\hline \multirow{2}{*}{ N. mediterraneum } & CBS 121718 & Eucalyptus sp. & Greece & GU251176 & GU251308 & GU251836 \\
\hline & CBS 121558 & Olea europaea & Italy & GU799463 & GU799462 & GU799461 \\
\hline \multirow[t]{2}{*}{ N. nonquaesitum } & CBS 126655 & Umbellularia & USA & GU251163 & GU251295 & GU251823 \\
\hline & PD 301 & Vaccinium corymbosum & Chile & GU251164 & GU251296 & GU251824 \\
\hline \multirow[t]{2}{*}{ N. occulatum } & CBS 128008 & Eucalyptus grandis hybrid & Australia & EU301030 & EU339509 & EU339472 \\
\hline & MUCC 286 & Eucalyptus pellita & Australia & EU736947 & EU339511 & EU339474 \\
\hline \multirow[t]{2}{*}{ N. parvum } & CMW 9081 & Actinidia deliciosa & New Zealand & AY236943 & AY236888 & AY236917 \\
\hline & CBS 110301 & Vitis vinifera & Portugal & AY259098 & AY573221 & EU673095 \\
\hline N. pennatisporum & MUCC 510 & Allocasuarina fraseriana & Australia & EF591925 & EF591976 & EF591959 \\
\hline N. pistaciae & CBS 595.76 & Pistacia vera & Greece & KX464163 & KX464676 & KX464953 \\
\hline \multirow[t]{2}{*}{ N. pistaciarum } & CBS 113083 & Pistacia vera & USA & KX464186 & KX464712 & KX464998 \\
\hline & CBS 113084 & redwood & USA & KX464187 & KX464713 & KX464999 \\
\hline
\end{tabular}




\begin{tabular}{|c|c|c|c|c|c|c|}
\hline N. protearum & CBS114176 & Protea sp. & South Africa & AF452539 & KX464720 & KX465006 \\
\hline \multirow[t]{2}{*}{ N. ribis } & CBS 115475 & Ribes sp. & USA & AY236935 & AY236877 & AY236906 \\
\hline & CBS 121.26 & R. rubrum & USA & AF241177 & AY236879 & AY236908 \\
\hline N. sinense & CGMCC 3.18315 & unknown woody plant & Guizhou,China & KY350148 & KY817755 & KY350154 \\
\hline \multirow[t]{2}{*}{ N. stellenboschiana } & CBS 110864 & Vitis vinifera & South Africa & - & - & KX465047 \\
\hline & CBS 282.70 & Arum italicum & Spain & KX464225 & KX464758 & KX465051 \\
\hline \multirow[t]{2}{*}{ N. terminaliae } & CMW 26679 & Terminalia sericea & South Africa & GQ471802 & GQ471780 & KX465052 \\
\hline & CMW 26683 & Terminalia sericea & South Africa & GQ471804 & GQ471782 & KX465053 \\
\hline \multirow[t]{2}{*}{ N. umdonicola } & CBS 123645 & Syzygium cordatum & South Africa & EU821904 & EU821874 & EU821844 \\
\hline & CBS 123646 & Syzygium cordatum & South Africa & EU821905 & EU821875 & EU821845 \\
\hline \multirow[t]{2}{*}{ N. ursorum } & CMW 24480 & Eucalyptus arboretum & South Africa & FJ752746 & FJ752709 & KX465056 \\
\hline & CMW 23790 & Eucalyptus arboretum & South Africa & FJ752745 & FJ752708 & KX465057 \\
\hline \multirow[t]{2}{*}{ N. viticlavatum } & CBS 112878 & Vitis vinifera & South Africa & AY343381 & AY343342 & KX465058 \\
\hline & CBS 112977 & Vitis vinifera & South Africa & AY343380 & AY343341 & KX465059 \\
\hline \multirow[t]{2}{*}{ N. vitifusiforme } & CBS 110887 & Vitis vinifera & South Africa & AY343383 & AY343343 & KX465061 \\
\hline & CBS 110880 & Vitis vinifera & South Africa & AY343382 & AY343344 & - \\
\hline Botryosphaeria corticis & CBS 119047 & Vaccinium corymbosum & USA & DQ299245 & EU017539 & EU673107 \\
\hline Botryosphaeria dothidea & CBS 115476 & Prunus sp. & Switzerland & AY236949 & AY236898 & AY236927 \\
\hline
\end{tabular}




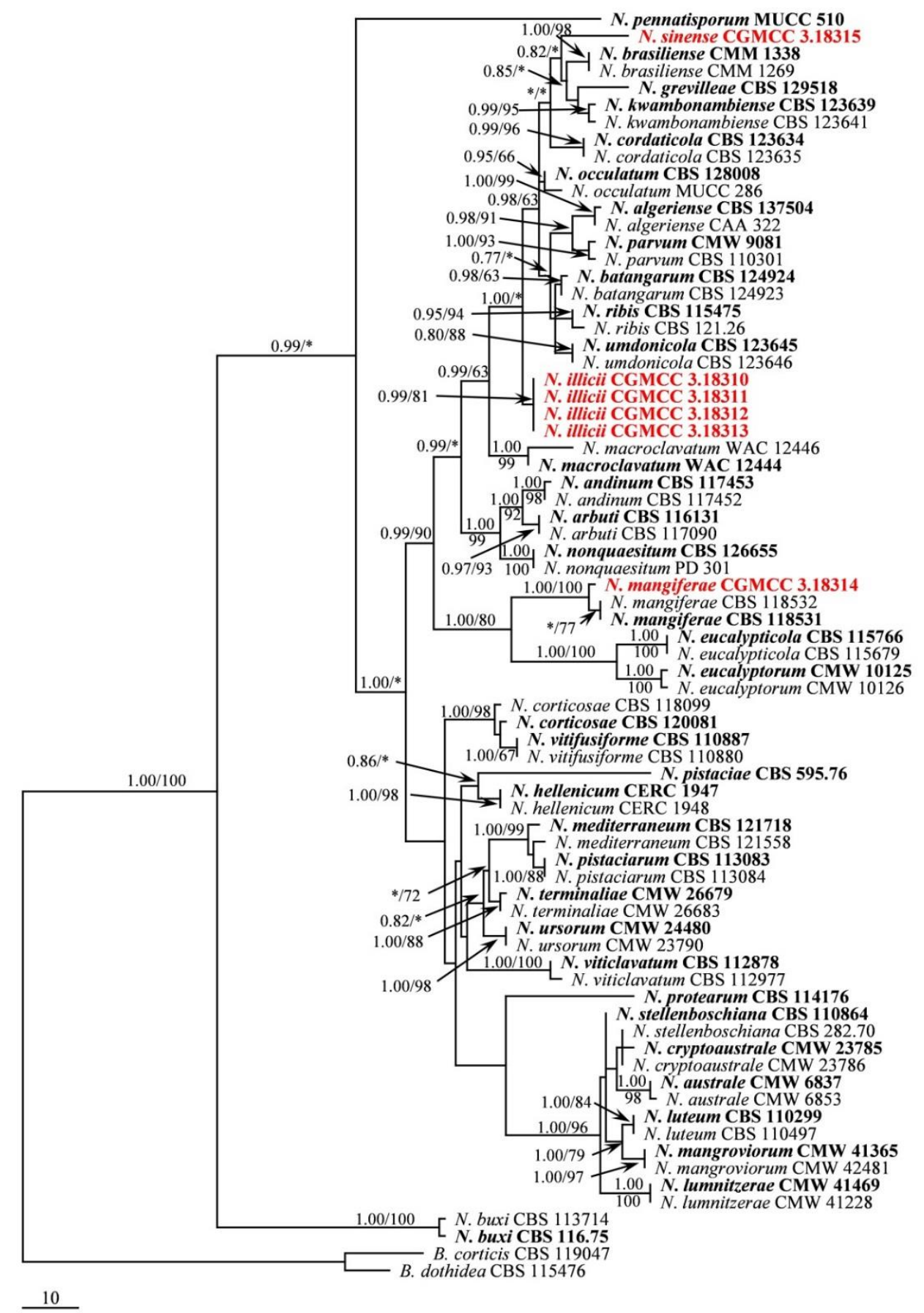

Figure 1 - Maximum parsimony tree obtained from combined ITS, tefl- $\alpha$ and TUB sequence data of Neofusicoccum species. The tree is rooted to Botryosphaeria corticis (CBS 119047) and B. dothidea (CBS 115476). Bayesian posterior probabilities (PP) support above $70 \%$ and maximum parsimony (MP) support values above 60 are shown with Bayesian PP followed by MP bootstrap (PP/MP) values at the nodes. Ex-type strains are printed in bold face and new isolates in red bold face. 

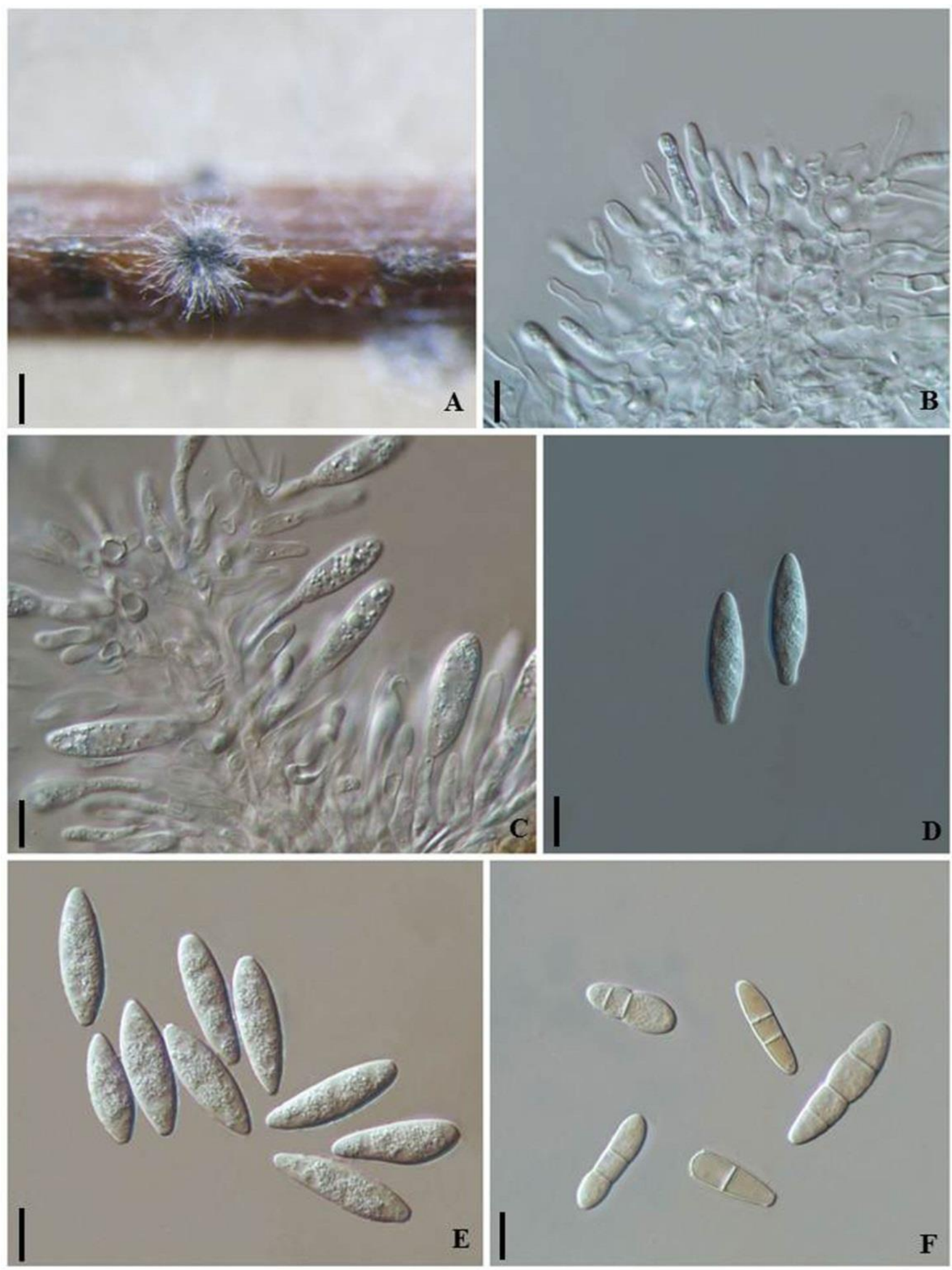

Figure 2 - Neofusicoccum illicii (from holotype: HMAS 266205). A. Conidiomata developing on pine needles in culture. B, C. Conidiogenous cells with developing conidia. D. Hyaline, aseptate conidia with a node-like base. E. Hyaline, aseptate conidia. F. Yellowish, 1-3-septate, senescent conidia. Scale bars: $\mathrm{A}=250 \mu \mathrm{m}, \mathrm{B}-\mathrm{F}=10 \mu \mathrm{m}$. 

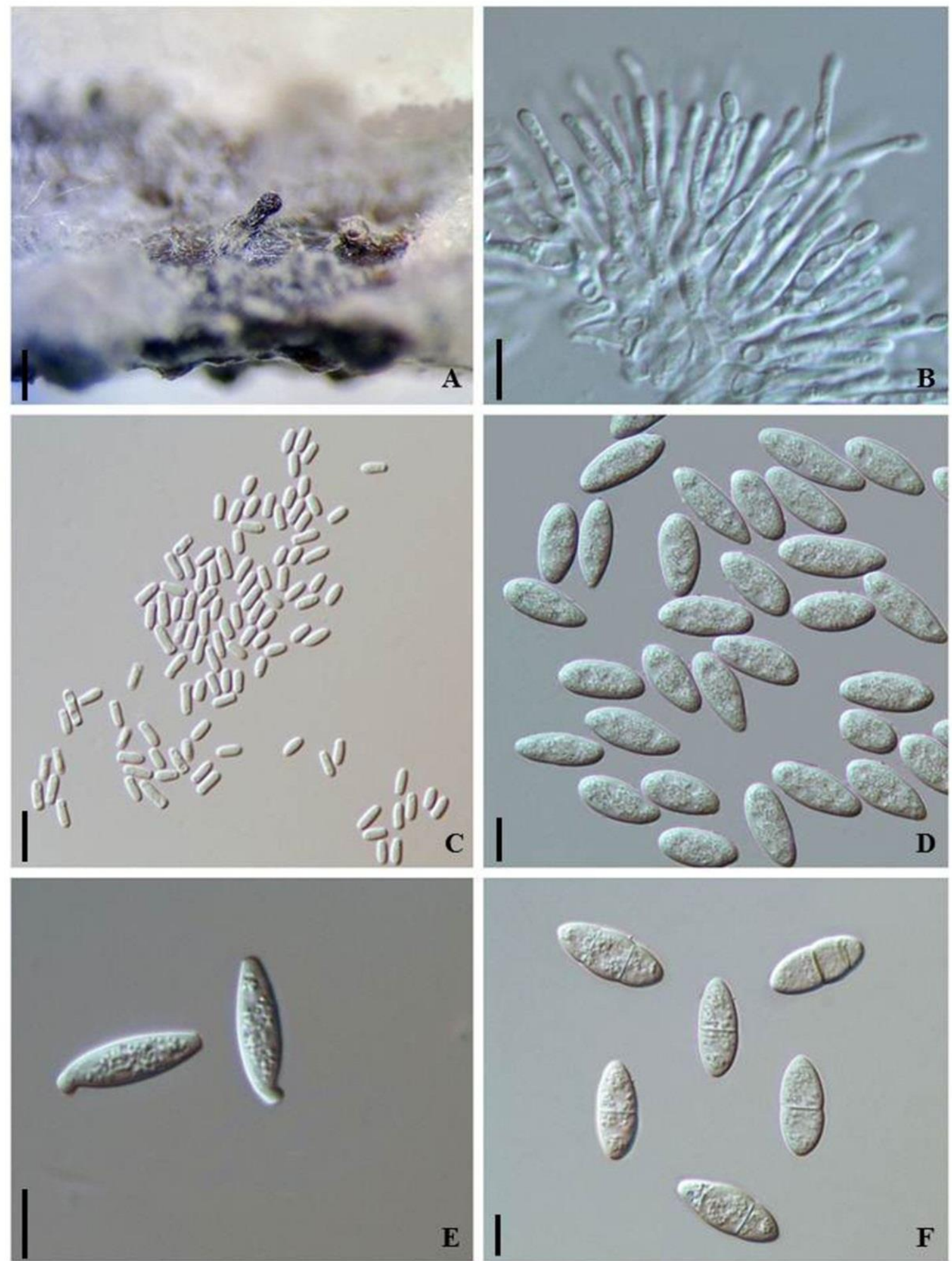

Figure 3 - Neofusicoccum sinense (from holotype: HMAS 255209). A. Conidiomata formed on pine needles in culture. B. Spermatogenous cells with developing spermatia. C. Spermatia. D. Hyaline, aseptate conidia. E. Hyaline, aseptate conidia with a curved node-like base. F. 1- or 2septate, hyaline, senescent conidia. Scale bars: $A=300 \mu \mathrm{m}, \mathrm{B}-\mathrm{F}=10 \mu \mathrm{m}$.

\section{Acknowledgements}

This study was supported by "the Fundamental Research Funds for the Central Universities (No. 2016ZCQ04)", NSFC Projects of International Cooperation and Exchanges (31461143028), National Natural Science Foundation of China (General Program, 31370063) and National Science and Technology Foundation Project (2014FY210400). 


\section{References}

Abdollahzadeh J, Zare R, Phillips AJL. 2013 - Phylogeny and taxonomy of Botryosphaeria and Neofusicoccum species in Iran, with description of Botryosphaeria scharifii sp. nov. Mycologia 105, 210-220.

Adesemoye AO, Eskalen A. 2011 - First report of Spencermartinsia viticola, Neofusicoccum austral \& N. parvum causing branch canker of Citrus in California. Plant Disease 95, 770.

Alves A, Crous PW, Correia A, Phillips AJL. 2008 - Morphological and molecular data reveal cryptic speciation in Lasiodiplodia theobromae. Fungal Diversity 28, 1-13.

Arx JA von 1987 - Plant-pathogenic Fungi. J. Cramer, Berlin, Germany.

Barradas C, Correia A, Alves A. 2013 - First report of Neofusicoccum australe and N. luteum associated with canker and dieback of Quercus robur in Portugal. Plant Disease 97, 560.

Begoude BAD, Slippers B, Wingfield MJ, Roux J. 2010 - Botryosphaeriaceae associated with Terminalia catappa in Cameroon, South Africa and Madagascar. Mycological Progress 9, 101-123.

Berraf-Tebbal A, Guereiro MA, Phillips AJL. 2014 - Phylogeny of Neofusicoccum species associated with grapevine trunk diseases in Algeria, with description of Neofusicoccum algeriense sp. nov. Phytopathologia Mediterranea 53, 416-427.

Crous PW, Groenewald JZ, Shivas RG, Edwards J et al. 2011 - Fungal Planet Description Sheets: 69-91. Persoonia 26, 108-156.

Crous PW, Slippers B, Wingfield MJ, Rheeder J et al. 2006 - Phylogenetic lineages in the Botryosphaeriaceae. Studies in Mycology 55, 235-253.

Dissanayake AJ, Phillips AJL, Li XH, Hyde KD. 2016 - Botryosphaeriaceae: Current status of genera and species. Mycosphere 7, 1001-1073.

Dissanayake AJ, Zhang W, Li XH, Zhou Y et al. 2015 - First report of Neofusicoccum mangiferae associated with grapevine dieback in China. Phytopathologia Mediterranea 54, 414-419.

Glass NL, Donaldson GC. 1995 - Development of primer sets designed for use with the PCR to amplify conserved genes from filamentous Ascomycetes. Applied and Environmental Microbiology 61, 1323-1330.

Huelsenbeck JP, Ronquist F. 2001 - MRBAYES: Bayesian inference of phylogenetic trees. Bioinformatics 17, 754-755.

Ismail AM, Cirvilleri G, Lombard L, Crous PW et al. 2013 - Characterisation of Neofusicoccum species causing mango dieback in Italy. Journal of Plant Pathology 95, 549-557.

Johnson GI, Cooke AW, Mead AJ, Wells IA. 1991 - Stem-end rot of mango in Australia: cause and control. Acta Horticulturae 291, 288-295.

Johnson GI. 1992 - Biology and control of stem-end rot pathogens of mango. [Doctoral dissertation]. Queensland, Australia: University of Queensland. 265 p.

Kong CS, Qiu XL, Yi KS, Yu XF et al. 2010 - First report of Neofusicoccum vitifusiforme causing blueberry blight of blueberry in China. Plant Disease 94, 1373.

Krishnapillai N, Wijeratnam RSW. 2015 - First report of Neofusicoccum mediterraneum causing stem end rot on Karuthakolumban mangoes. Plant Disease 99, 1858-1858.

Li QL, Deng TJ, Huang SP, Guo TX et al. 2014 - First report of gummosis of Mango trees caused by Neofusicoccum parvum in Sichuan, Southwest China. Journal of Plant Pathology 96, 113131.

Li QL, Guo TX, Pan ZB, Huang SP et al. 2013 - An outbreak of gummosis of mango trees caused by Lasiodiplodia theobromae in Guangxi, south China. Plant Disease 97, 690.

Lopes UP, Zambolim L, Pinho DB, Barros AV et al. 2014 - Postharvest rot and mummification of strawberry fruits caused by Neofusicoccum parvum and N. kwambonambiense in Brazil. Tropical Plant Pathology 39, 178-183.

Marques MW, Lima NB, de Morais MA Jr, Michereff SJ et al. 2013 - Botryosphaeria, Neofusicoccum, Neoscytalidium and Pseudofusicoccum species associated with mango in Brazil. Fungal Diversity 61, 195-208. 
Mitra SK, Baldwin EA. 1997 - Mango. In: Mitra MA, ed. Postharvest physiology and storage of tropical and subtropical fruits. Oxon, United Kingdom: Center for Agriculture and Biosciences International, 85-122.

Page RD. 1996 - TreeView: an application to display phylogenetic trees on personal computers. Computer Applications in the Biosciences 12, 357-358.

Pavlic D, Wingfield MJ, Barber P, Slippers B et al. 2008 - Seven new species of the Botryosphaeriaceae from baobab and other native trees in Western Australia. Mycologia 100, 851-866.

Phillips AJL, Alves A, Pennycook SR, Johnston PR et al. 2008 - Resolving the phylogenetic and taxonomic status of dark-spored teleomorph genera in the Botryosphaeriaceae. Persoonia 21, $29-55$.

Posada D, Buckley TR. 2004 - Model selection and model averaging in phylogenetics: advantages of Akaike information criterion and Bayesian approaches over likelihood ratio tests. Systematic Biology 53, 793-808.

Ronquist F, Huelsenbeck JP. 2003 - MrBayes 3: Bayesian phylogenetic inference under mixed models. Bioinformatics 19, 1572-1574.

Sakalidis ML, Hardy GEStJ, Burgess TI. 2011 - Use of the genealogical sorting index (GSI) to delineate species boundaries in the Neofusicoccum parvum- Neofusicoccum ribis species complex. Molecular Phylogenetics and Evolution 60, 333-344.

Serrato-Diaz LM, Rivera-Vargas L, French-Monar RD. 2014 - First Report of Neofusicoccum mangiferae Causing Rachis Necrosis and Inflorescence Blight of Mango (Mangifera indica) in Puerto Rico. Plant Disease 98, 570-571.

Slippers B, Crous PW, Denman S, Coutinho TA et al. 2004 - Combined multiple gene genealogies and phenotypic characters differentiate several species previously identified as Botryosphaeria dothidea. Mycologia 96, 83-101.

Slippers B, Johnson GI, Crous PW, Coutinho TA et al. 2005 - Phylogenetic and morphological reevaluation of the Botryosphaeria species causing diseases of Mangifera indica. Mycologia 97, 99-110.

Swofford DL. 2002 - PAUP*. Phylogenetic Analysis Using Parsimony (*and Other Methods). Version 4. Sinauer Associates, Sunderland, Massachusetts.

Sydow H, Sydow P, Butler EJ. 1916 - Fungi Indiae Orientalis. V. Annalles Mycologici 14, 177220.

Tamura K, Stecher G, Peterson D, Filipski A et al. 2013 - MEGA6: molecular evolutionary genetics analysis version 6.0. Molecular Biology \& Evolution 30, 2725-2729.

White TJ, Bruns T, Lee S, Taylor J. 1990 - Amplification and direct sequencing of fungal ribosomal RNA genes for phylogenetics. In: PCR Protocols: a guide to methods and applications (Innes MA, Gelfand DH, Snisky JJ, White TJ, eds.) Academic Press, San Diego, California: 315-322.

Yu L, Impaprasert R, Zhao JR, Xu SG et al. 2013 - Stem die-back of highbush blueberries caused by Neofusicoccum parvum in China. New Disease Reports 27, 3.

Yu ZD, Tang GH, Peng SB, Chen H et al. 2015 - Neofusicoccum parvum causing canker of seedlings of Juglans regia in China. Journal of Forest Research 26, 1019-1024.

Zhang Y, Wang HK, Fournier J, Crous PW et al. 2009 - Towards a phylogenetic clarification of Lophiostoma / Massarina and morphologically similar genera in the Pleosporales. Fungal Diversity 38, 225-251. 